\title{
Antibiotic Microbial Prophylaxis for Spinal Surgery: Comparison between 48 and 72-Hour AMP Protocols
}

\author{
Boram Kim, Seong-Hwan Moon, Eun-Su Moon, Hak-Sun Kim, \\ Jin-Oh Park, In-Je Cho, Hwan-Mo Lee \\ Department of Orthopedic Surgery, Yonsei University College of Medicine, Seoul, Korea
}

\begin{abstract}
Study Design: This is a prospective randomized cohort study.
Purpose: We intended to evaluate the efficacy of a 48 hour antibiotic microbial prophylaxis (AMP) protocol as compared with a 72 hour AMP protocol.

Overview of Literature: The current guideline for the prevention of surgical site infection (SSI) suggests the AMP should not exceed 24 hours after clean surgery like spinal surgery. But there exist some confusion in real clinical practice about the duration of postoperative antibiotic administration because the evidence of the guideline was not robust.

Methods: The subjects were 548 patients who underwent spinal surgery at our department from April 2007 to December 2008. The patients were classified into two groups according to the prophylaxis protocol: group A, for which AMP was employed for 72 hours postoperatively and group B, for which AMP was employed for 48 hours postoperatively. Five hundred two patients out of 548 patients were followed until 6 months postoperatively. The incidence of SSI in the two groups was analyzed.
\end{abstract}

Results: The overall infection rate was $0.8 \%$. There was no significant difference in infection rate between the two groups. The overall infection rate for the patients who underwent instrumented fusion was $0.9 \%$. There was no significant difference in the infection rate between the patients of the two groups who underwent instrumented fusion.

Conclusions: AMP for 48 hours is as efficient as AMP for 72 hours.

Key Words: Spine, Surgical site infection, Anti-bacterial agents

\section{Introduction}

Surgical site infection (SSI) is one of the major complications after spinal surgery [1]. Because it is a devastating complication despite of its low incidence, there has been much effort to prevent postoperative infection. Antibiotic microbial prophylaxis (AMP) is one of major modalities to prevent this malady.

It is well known that the infection rate of a group that received prophylactic antibiotics was significantly lower than that of a group that did not receive prophylactic antibiotics when undergoing elective orthopedic surgery, including spinal surgery $[2,3]$. Many studies have shown the effectiveness of AMP in spinal surgery [4-7].

Regarding the dose of antibiotics, many studies also have shown that there was no additional benefit with a multiple dose regimen [8]. The current consensus is that antibiotic administration should be started within 30 to 60 minutes before skin incision. The current guideline for the preven-

Received Feb 1, 2010; 1st Revised Apr 1, 2010; Accepted Apr 2, 2010

Corresponding author: Hwan-Mo Lee, MD

Department of Orthopedic Surgery, Yonsei University College of Medicine,

134 Sinchon-dong, Seodaemun-gu, Seoul 120-752, Korea

Tel: +82-2-2228-21, Fax: +82-2-363-113, E-mail: hwanlee@yuhs.ac 
tion of SSI that was published by the Center for Disease Control and Prevention also suggests that postoperative antibiotics should be discontinued within 24 hours after clean surgery, including spinal surgery [1,9]. But there exist some confusion in real clinical situations about the proper duration of postoperative antibiotic administration because the evidence of the guideline was not robust.

Spinal surgery is classified as clean surgery [10] that doesn't need additional AMP for more than 24 hours postoperatively, according to the guideline [11]. However, basic research and clinical research has shown that surgery with instrumentation has a higher risk of infection than that of non-instrumented surgery [12-14], which might suggest that a different AMP protocol is needed for instrumented proce-

Table 1. Demographic and surgical characteristics of patients

\begin{tabular}{lc}
\hline \hline Characteristic & No. of patients $(\%)$ \\
\hline Age (mean [range], yr) & $59.1(16-86)$ \\
Sex (female) & $344(62.2)$ \\
Type of surgery & \\
Laminectomy only (including laminoplasty) & $58(10.5)$ \\
Discectomy with laminectomy & $141(25.5)$ \\
Arthrodesis with instrumentation & $345(62.4)$ \\
Instrumentation only & $9(1.6)$ \\
Operation region & \\
Cervical & $32(5.8)$ \\
Thoracic & $24(4.3)$ \\
Lumbar & $497(89.9)$ \\
\hline
\end{tabular}

dures compared with non-instrumented procedures. It is not uncommon to use antibiotics for 72 hours after spinal surgery even in the tertiary institutes in Korea.

At our institute, we also administered prolonged antibiotics for 72 hours postoperatively before 2007. But we changed our protocol and we have been administering antibiotics for 48 hours or 72 hours postoperatively since 2007.

This study purposes to determine the effectiveness of a 48 hour antibiotic prophylaxis protocol compared with that of a 72 hour antibiotic prophylaxis protocol.

\section{Materials and Methods}

The patients who were older than 15 years old and had undergone clean spinal surgery by two orthopedic spinal surgeons (LHM, MSH) were consecutively recruited from April 2007 to December 2008. A total of 548 patients were recruited.

From April 2007, two orthopedic spinal surgeons in our institute have used two different prophylaxis protocols in all their orthopedic spinal surgery. One used the protocol in which the preoperative antibiotics start within 30 minutes before skin incision and the antibiotics are administered until 72 hours postoperatively (protocol A). Another protocol is that the preoperative antibiotics start within 30 minutes before skin incision and the antibiotics are adminis-

Table 2. Characteristics of two groups

\begin{tabular}{|c|c|c|c|}
\hline \multirow{2}{*}{ Characteristics } & \multicolumn{2}{|c|}{ No. of patients (\%) } & \multirow{2}{*}{$p$-value } \\
\hline & Group A $(n=281)$ & Group B $(n=221)$ & \\
\hline Mean age (yr) & 58.8 & 60.4 & $0.211^{\mathrm{a})}$ \\
\hline Body mass index & 23.97 & 24.32 & $0.252^{\mathrm{a})}$ \\
\hline Sex (female) & $173(61.6)$ & $145(65.6)$ & $0.350^{\mathrm{b})}$ \\
\hline Diabetes mellitus & $47(9.4)$ & $47(21.3)$ & $0.195^{\mathrm{b})}$ \\
\hline Steroid use & $13(4.6)$ & $8(3.6)$ & $0.576^{\mathrm{b})}$ \\
\hline Immunosuppressive agent use & $9(3.2)$ & $5(2.3)$ & $0.525^{\mathrm{b})}$ \\
\hline Smoking & $13(4.6)$ & $9(4.1)$ & $0.763^{\mathrm{b})}$ \\
\hline Alcohol abuse & $9(3.2)$ & $8(3.6)$ & $0.798^{\mathrm{b})}$ \\
\hline Operating region & & & $0.151^{\mathrm{c}}$ \\
\hline Cervical & $19(6.8)$ & $10(4.5)$ & \\
\hline Thoracic & $8(2.8)$ & $10(4.5)$ & \\
\hline Lumbar & $247(87.9)$ & $200(90.5)$ & \\
\hline Thoracolumbar & $7(2.5)$ & $1(0.5)$ & \\
\hline Multilevel surgery ${ }^{\mathrm{d})}$ & $10(3.6)$ & $14(6.3)$ & $0.148^{\mathrm{b})}$ \\
\hline Instrumented arthrodesis ${ }^{\mathrm{e})}$ & $130(46.3)$ & $194(87.8)$ & $<0.05^{\mathrm{b})}$ \\
\hline Revisional operation & $4(1.4)$ & $2(0.9)$ & $0.699^{\mathrm{c}}$ \\
\hline
\end{tabular}

${ }^{\mathrm{a}}$ From t-test, ${ }^{\mathrm{b}}$ From Pearson chi-square test, ${ }^{\mathrm{c}}$ From Fisher's exact test, ${ }^{\mathrm{d})}$ Three level or more, ${ }^{\mathrm{e}}$ Statistically significant. 
tered until 48 hours postoperatively (protocol B). Another shot of antibiotics was injected if the time of the procedure had exceeded 4 hours or the blood loss was over 1,000 ml intraoperatively for both protocols. The Foley catheter was routinely removed within 48 hours. There were 301 patients (54.9\%) in group A and 247 patients (45.1\%) in group B. First generation cephalosporins were used as prophylactic antibiotics.

The demographic and clinical characteristics that had been previously reported as risk factors for SSI were recorded. The demographic information and surgical characteristics of the patients are summarized in Table 1. The patients were followed until 6 months postoperatively to detect surgical site infection.

Comparison between the two groups was performed by the t-test for continuous variables and the chi-square test for categorical variables. The difference in the infection rate between the two groups was determined by Fisher's exact test.

\section{Results}

Forty six patients of the 548 patients $(8.4 \%)$ were not followed up until 6 months after surgery. The number of patients who were not followed in groups A and B was 20 and 26, respectively. Five hundred two patients of the 548 patients were followed until 6 months after surgery. Among them, there were 281 patients in group A and 221 patients

Table 3. Infection rate

\begin{tabular}{lccc}
\hline \hline Group & No. of infection (\%) & No. of non-infection & Total \\
\hline A & $1(0.4)$ & 280 & 281 \\
B & $3(1.4)$ & 221 & 221 \\
Total & $4(0.8)$ & 498 & 502 \\
\hline
\end{tabular}

in group B.

The mean age of the group A and group B patients was 58.8 and 60.4 , respectively. There was no significant difference between the two groups $(p=0.211)$. The other variables of the two groups are shown in Table 2. There were no significant difference between the two groups regarding each variable, except for instrumentation and bone grafting. The ratio of instrumented fusion to total surgery was higher in group $\mathrm{B}$ and the difference of the ratios between the two groups was statistically significant $(p<0.01)$.

The overall incidence of SSI was $0.8 \%$ (4 of 502) (Table $3)$. The infection rate of group A was $0.4 \%$ ( 1 of 281). The infection rate of group B was 1.4\% (3 of 221). There was no significant difference between the two groups $(p=0.325)$ according to Fisher's exact test.

All the infections happened in the patients who received lumbar instrumentation (three cases of instrumented lumbar fusion; one case of lumbar instrumentation). Two of the four cases of infection were classified as superficial incisional; one case was classified as deep incisional infection and 1 case was classified as organ space infection. Methicilin-resistant Staphylococcous aureus was isolated in all 4 cases (Table 4).

We performed a sub-analysis for the cases with instrumented fusion (Table 5). The total number of cases with instrumented fusion was 318 (127 in group A and 191 in group B). The overall infection rate was $0.9 \%$ (3 of 318). The infection rate of group A was $0.8 \%$ (1 of 127). The infection rate of group B was 1.0\% (2 of 191). There was no significant difference of the infection rate between the two groups $(p=1.000)$ according to Fisher's exact test. In contrast, there was no infection among the 168 cases (147 in group A; 21 in group B) that underwent decompression without instrumentation or discectomy.

Table 4. Detail information of infection cases

\begin{tabular}{|c|c|c|c|c|c|c|c|}
\hline Case & Group & Age/Sex & Diagnosis & Operation & Cormobidity & Category & Organism \\
\hline 1 & A & 68/M & Spinal stenosis, L3-4-5 & $\begin{array}{l}\text { Decompression and } \\
\text { instrumented fusion }\end{array}$ & $\begin{array}{l}\mathrm{S} / \mathrm{P} \text { pituitary } \\
\text { adenoma }\end{array}$ & Organ space & MRSA \\
\hline 2 & B & $50 / \mathrm{M}$ & Metastatic spinal tumor, T11 & $\begin{array}{l}\text { Decompression and } \\
\text { instrumentation }\end{array}$ & $\mathrm{RCC}$ & $\begin{array}{l}\text { Superficial } \\
\text { incisional }\end{array}$ & MRSA \\
\hline 3 & B & $67 / F$ & Spondylolisthesis L4 & PLIF and instrumentation & None & Deep incisional & MRSA \\
\hline 4 & B & $53 / \mathrm{M}$ & Spinal stenosis, L3-4-5 & $\begin{array}{l}\text { Decompression and } \\
\text { instrumented fusion }\end{array}$ & None & $\begin{array}{l}\text { Superfical } \\
\text { incisional }\end{array}$ & MRSA \\
\hline
\end{tabular}

M: Male, F: Female, S/P: Postoperative status, MRSA: Methicilin-resistant Staphylococcus aureus, RCC: Renal cell carcinoma, PLIF: Posterior lumbar interbody fusion. 
Table 5. Infections in instrumented fusion group

\begin{tabular}{lccc}
\hline \hline Group & No. of infection (\%) & No. of non-infection & Total \\
\hline Group A & $1(0.8)$ & 126 & 127 \\
Group B & $2(1.0)$ & 189 & 191 \\
Total & $3(0.9)$ & 315 & 318 \\
\hline
\end{tabular}

\section{Discussion}

SSI is diagnosis by the clinical signs and symptoms such as fever, pain or purulent discharge on the operative site, and the image findings. SSI is categorized into superficial incisional infection, deep incisional infection and organ space infection according to the Center for Disease Control and Prevention guideline in 1999 [10]. Superficial incisional infection was defined as infection above the lumbodorsal fascia and deep incisional infection was defined as infection deep to the fascia. Organ space infection was defined as discitis or osteomyelitis of the vertebra.

The main cause of SSI following operations is bacterial contamination of the surgical site during procedures [15]. However, only a small amount of bacterial contamination can't cause infection. It takes contamination with a substantial number of bacteria to cause infection. Krizek and Robson [16] demonstrated that contamination with more than $10^{5}$ bacteria significantly increased the risk of wound infection. The role of antibiotic prophylaxis is to control the number of contaminating bacteria below the significant level that cannot cause infection, and not to eradicate all the bacteria. Accordingly, the concentration of antibiotics should be at the maximum when the skin is incised to control bacteria that are colonized on skin. To achieve this, the antibiotics should be administered within 30 to 60 minutes before skin incision $[11,17]$.

Although preoperative antibiotic prophylaxis is effective to prevent bacterial infection, prolonged use of antibiotics cannot be justified. A meta-analysis by Barker [8] didn' t identify any additional benefit of multiple-dose regimens. Prolonged antibiotic use increases the risk of resistant strains without any benefit [18]. In addition, the current guideline for clean surgery is single dose antibiotic prophylaxis and if another dose is added, it should not exceed 24 hours postoperatively $[10,11]$.

Despite of this consensus, the evidence for single dose antibiotic prophylaxis is not robust, and especially for orthopedic surgery with an implant [19]. Because a smaller number of bacteria can cause infection with foreign material
[10], clean surgery with implantation has more of a risk for infection. In addition, it might be appropriate to increase the duration of prophylactic antibiotics for high risk patients [20].

All this might cause confusion in the current clinical situation. For example, in Korea, the usual duration of prophylactic antibiotics is more than 72 hours. We adopted our current prophylactic antibiotics protocol since 2007 in our institute. We adopted a 72 hour protocol and a 48 hour protocol for AMP to reduce antibiotic usage.

The results of this study showed that there was no significant difference of the infection rate between the 48 hour group and the 72 hour group. The two groups were consecutively recruited, which is a popular method for conducting clinical research to minimize a selection bias. However, when the clinical characteristics of the 2 groups were compared, the proportions of instrumented fusion of the two groups were significantly different. We performed a subanalysis for the cases with instrumented fusion because instrumented fusion might contribute to an increase risk for infection [11,12,21].

Various clinical characteristics have previously been reported as risk factors for surgical site infection. Olsen et al. [14] stated that diabetes was the highest independent risk factor for spinal SSI in their recent study. Suboptimal timing of prophylactic antibiotic therapy was another important risk factor in their study. We routinely gave prophylactic antibiotics just after positioning the patient, which was less than 30 minutes before skin incision. However, we didn' t check the exact time of administration.

Fang et al. [20] showed that an advanced age, smoking, diabetes, previous surgical infection, an increased body mass index and alcohol abuse were risk factors for SSI. In addition, instrumentation, steroid use, immunosupression, multilevel surgery, lumbar surgery and revision operation have also been reported as risk factors [10-12].

Many studies have reported that the infection rate of posterior spinal fusion with instrumentation was higher than that for other procedures. Massie et al. [12] reported that the infection rate of instrumented fusion was $6 \%$ or more. In contrast, the infection rate of discectomy was less than $1 \%$ and it was 1-5\% for spinal fusion without instrumentation in their systemic review [12]. Perry et al. [21] reported a 9.7\% infection rate after segmental spinal instrumentation, which was higher than the usual infection rate after spinal surgery, and Wimmer et al. [22] reported that most infections occurred with posterior instrumentation in their study. In 
our study, all the infections were associated with posterior instrumentation and 3 of the 4 infection cases were related with posterior instrumented fusion. This result validates that posterior instrumentation and instrumented fusion were major risk factors for spinal SSI and it might suggest that instrumented spinal surgery might have to be dealt with in a different way in regard to AMP.

The overall infection rate after spinal surgery has ranged from $0.6 \%$ to $12.7 \%$ according to the previous studies [2$4,8,11,13,14,18,23,24]$ The reported infection rate following posterior spinal fusion with instrumentation has ranged from $2.1 \%$ to $6 \%[5,12,14]$. This study showed a consistent result for the overall infection rate $(0.8 \%)$ and it showed a lower infection rate after instrumented fusion $(0.9 \%)$ than previsous studies, which was not statistically determined.

This study has some limitations. First of all, the sample size was relatively small. A large sample is needed for strong statistical power, but this is difficult to achieve as the incidence of SSI is very low. Dimick et al. [25] determined that 5,036 patients would need to be enrolled to demonstrate a 50\% reduction in the infection rate, based on the assumption that a wound infection rate of $2 \%$ is typical for spinal surgery. The sample of this study also contained a small number of high risk patients, such as patients with diabetes mellitus, steroid use and smoking, which might impair the ability to generalize the results of this sample to a larger population.

The second limitation is that the two groups, which had different antibiotic prophylaxis protocols, underwent operation from different surgeons, although consecutive recruitment was done.

The ratio of the types of procedures was different. The ratio of the cases with instrumented fusion, which is a major risk factor for surgical site infection, was higher in group B. Accordingly, we performed a sub-analysis for the cases with instrumented fusion, and there was no significant difference between the two groups. Future studies with larger size samples are needed to confirm our results.

\section{Conclusions}

For clean spine surgery, AMP for 48 hours is as efficient as AMP for 72 hours.

\section{REFERENCES}

1. Carreon LY, Puno RM, Dimar JR 2nd, Glassman SD, Johnson JR. Perioperative complications of posterior lumbar decompression and arthrodesis in older adults. J Bone Joint Surg Am 2003;85:2089-92.

2. Pavel A, Smith RL, Ballard A, Larson IJ. Prophylactic antibiotics in elective orthopedic surgery: a prospective study of 1,591 cases. South Med J 1977;70 Suppl 1:50-5.

3. Piotrowski WP, Krombholz MA, Mühl B. Spondylodiscitis after lumbar disk surgery. Neurosurg Rev 1994;17:189-93.

4. Rubinstein E, Findler G, Amit P, Shaked I. Perioperative prophylactic cephazolin in spinal surgery: a double-blind placebo-controlled trial. J Bone Joint Surg Br 1994;76:99102.

5. Glassman SD, Dimar JR, Puno RM, Johnson JR. Salvage of instrumental lumbar fusions complicated by surgical wound infection. Spine (Phila Pa 1976) 1996;21:2163-9.

6. Rimoldi RL, Haye W. The use of antibiotics for wound prophylaxis in spinal surgery. Orthop Clin North Am 1996; 27:47-52.

7. Guiboux JP, Ahlgren B, Patti JE, Bernhard M, Zervos M, Herkowitz HN. The role of prophylactic antibiotics in spinal instrumentation: a rabbit model. Spine (Phila Pa 1976) 1998;23:653-6.

8. Barker FG 2nd. Efficacy of prophylactic antibiotic therapy in spinal surgery: a meta-analysis. Neurosurgery 2002;51: 391-400.

9. Herkowitz HN, Garfin SR, Eismont FJ, Bell GR, Balderston RA. Rothman-Simeone, the spine. 5th ed. Philadelphia: Saunders Elsevier, 2006.

10. Mangram AJ, Horan TC, Pearson ML, Silver LC, Jarvis WR. Guideline for Prevention of Surgical Site Infection, 1999: Centers for Disease Control and Prevention (CDC) Hospital Infection Control Practices Advisory Committee. Am J Infect Control 1999;27:97-132.

11. Brown EM, Pople IK, de Louvois J, et al. Spine update: prevention of postoperative infection in patients undergoing spinal surgery. Spine (Phila Pa 1976) 2004;29:938-45.

12. Massie JB, Heller JG, Abitbol JJ, McPherson D, Garfin SR. Postoperative posterior spinal wound infections. Clin Orthop Relat Res 1992;(284):99-108.

13. Levi AD, Dickman CA, Sonntag VK. Management of postoperative infections after spinal instrumentation. J Neurosurg 1997;86:975-80.

14. Olsen MA, Nepple JJ, Riew KD, et al. Risk factors for surgical site infection following orthopaedic spinal operations. 
J Bone Joint Surg Am 2008;90:62-9.

15. Rohde V, Meyer B, Schaller C, Hassler WE. Spondylodiscitis after lumbar discectomy: incidence and a proposal for prophylaxis. Spine (Phila Pa 1976) 1998;23:615-20.

16. Krizek TJ, Robson MC. Evolution of quantitative bacteriology in wound management. Am J Surg 1975;130:579-84.

17. Bratzler DW, Houck PM; Surgical Infection Prevention Guideline Writers Workgroup. Antimicrobial prophylaxis for surgery: an advisory statement from the National Surgical Infection Prevention Project. Am J Surg 2005;189:395404.

18. Kanayama M, Hashimoto T, Shigenobu K, Oha F, Togawa D. Effective prevention of surgical site infection using a Centers for Disease Control and Prevention guidelinebased antimicrobial prophylaxis in lumbar spine surgery. $\mathrm{J}$ Neurosurg Spine 2007;6:327-9.

19. AlBuhairan B, Hind D, Hutchinson A. Antibiotic prophylaxis for wound infections in total joint arthroplasty: a systematic review. J Bone Joint Surg Br 2008;90:915-9.
20. Fang A, Hu SS, Endres N, Bradford DS. Risk factors for infection after spinal surgery. Spine (Phila Pa 1976) 2005;30:1460-5.

21. Perry JW, Montgomerie JZ, Swank S, Gilmore DS, Maeder $\mathrm{K}$. Wound infections following spinal fusion with posterior segmental spinal instrumentation. Clin Infect Dis 1997; 24:558-61

22. Wimmer C, Gluch H, Franzreb M, Ogon M. Predisposing factors for infection in spine surgery: a survey of 850 spinal procedures. J Spinal Disord 1998;11:124-8.

23. Pilgaard S. Discitis (closed space infection) following removal of lumbar inervertebral disc. J Bone Joint Surg Am 1969;51:713-6.

24. Puranen J, Mäkelä J, Lähde S. Postoperative intervertebral discitis. Acta Orthop Scand 1984;55:461-5.

25. Dimick JB, Lipsett PA, Kostuik JP. Spine update: antimicrobial prophylaxis in spine surgery: basic principles and recent advances. Spine (Phila Pa 1976) 2000;25:2544-8. 